University of New Hampshire

University of New Hampshire Scholars' Repository

Space Science Center

Institute for the Study of Earth, Oceans, and

Space (EOS)

8-30-2006

\title{
Characterization of single-sided charge-sharing CZT strip detectors for gamma-ray astronomy
}

\author{
B Donmez \\ University of New Hampshire - Main Campus \\ John R. Macri \\ University of New Hampshire - Main Campus, John.Macri@unh.edu \\ James M. Ryan \\ University of New Hampshire, James.Ryan@unh.edu \\ Jason S. Legere \\ University of New Hampshire, jslegere@unh.edu \\ Mark L. McConnell \\ University of New Hampshire - Main Campus, mark.mcconnell@unh.edu
}

See next page for additional authors

Follow this and additional works at: https://scholars.unh.edu/ssc

Part of the Astrophysics and Astronomy Commons

\section{Recommended Citation \\ Burçin Dönmez ; John R. Macri ; James M. Ryan ; Jason S. Legere ; Mark L. McConnell ; Mark Widholm ; Tomohiko Narita and Louis-André Hamel "Characterization of single-sided charge-sharing CZT strip detectors for gamma-ray astronomy", Proc. SPIE 6319, Hard X-Ray and Gamma-Ray Detector Physics and Penetrating Radiation Systems VIII, 63190A (August 30, 2006); doi:10.1117/12.683695; http://dx.doi.org/ $10.1117 / 12.683695$}

This Conference Proceeding is brought to you for free and open access by the Institute for the Study of Earth, Oceans, and Space (EOS) at University of New Hampshire Scholars' Repository. It has been accepted for inclusion in Space Science Center by an authorized administrator of University of New Hampshire Scholars' Repository. For more information, please contact Scholarly.Communication@unh.edu. 


\section{Authors}

B Donmez, John R. Macri, James M. Ryan, Jason S. Legere, Mark L. McConnell, Mark Widholm, T Narita, and $L$ A. Hamel 


\title{
Characterization of Single-Sided Charge-Sharing CZT Strip Detectors for Gamma-Ray Astronomy
}

\author{
Burçin Dönmez ${ }^{a}$, John R. Macri ${ }^{a}$, James M. Ryan ${ }^{a}$, Jason S. Legere ${ }^{a}$, Mark L. McConnell ${ }^{a}$, \\ Mark Widholm $^{a}$, Tomohiko Narita ${ }^{b}$, Louis-André Hamel ${ }^{c}$ \\ ${ }^{a}$ Space Science Center, University of New Hampshire, 39 College Road, Durham, NH USA \\ 03824 ; \\ ${ }^{b}$ Dept. of Physics, College of the Holy Cross, 1 College Street, Worcester, MA USA 01610; \\ ${ }^{c}$ Dept. of Physics, University of Montreal, P.O. Box 6128 Downtown Station, Montreal, \\ Quebec, Canada, H3C 3J7
}

\begin{abstract}
We report progress in the study of thick single-sided charge-sharing cadmium zinc telluride (CZT) strip detector modules designed to perform spectroscopy and 3-D imaging of gamma-rays. We report laboratory measurements including spectroscopy, efficiency and 3-D imaging capability of prototype detectors $\left(15 \times 15 \times 7.5 \mathrm{~mm}^{3}\right)$ with $11 \times 11$ unit cells. We also report on Monte Carlo simulations (GEANT4 v7.1) to investigate the effect of multihits on detector performance in both spectroscopy and imaging. We compare simulation results with data obtained from laboratory measurements and discuss the implications for future strip detector designs.
\end{abstract}

Keywords: CZT, strip detectors, gamma-ray

\section{SINGLE-SIDED CHARGE-SHARING CZT STRIP DETECTORS}

\subsection{Detector Concept}

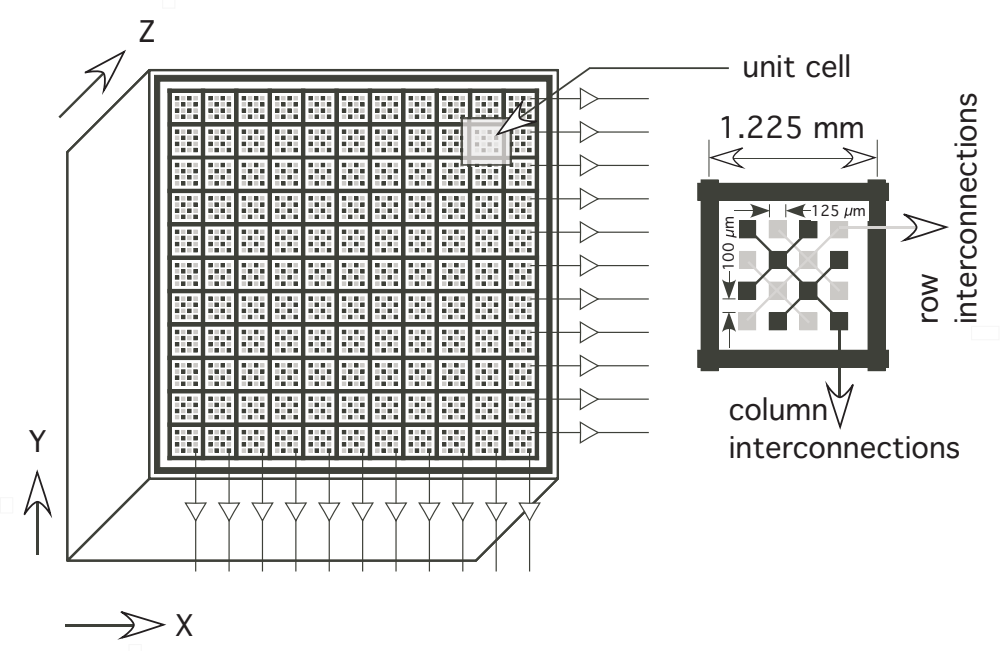

Figure 1. Single-sided charge-sharing strip detector (left). Unit cell (right) shows interconnections.

Figure 1 shows the single-sided charge-sharing CZT strip detector. ${ }^{1}$ This device has eleven row channels and eleven column channels (121 "pixels" or "unit cells"). The anode pattern and readout is shown. A $1.225 \mathrm{~mm}$

Further author information: (Send correspondence to B.D.)

B.D.: E-mail: bdonmez@comptel.sr.unh.edu, Telephone: 16038622756.

Hard X-Ray and Gamma-Ray Detector Physics and Penetrating Radiation Systems VIII,

edited by Larry A. Franks, Arnold Burger, Ralph B. James, H. Bradford Barber, F. Patrick Doty, Hans Roehrig,

Proc. of SPIE Vol. 6319, 63190A, (2006) $\cdot 0277-786$ X/06/\$15 $\cdot$ doi: 10.1117/12.683695

Proc. of SPIE Vol. 6319 63190A-1 


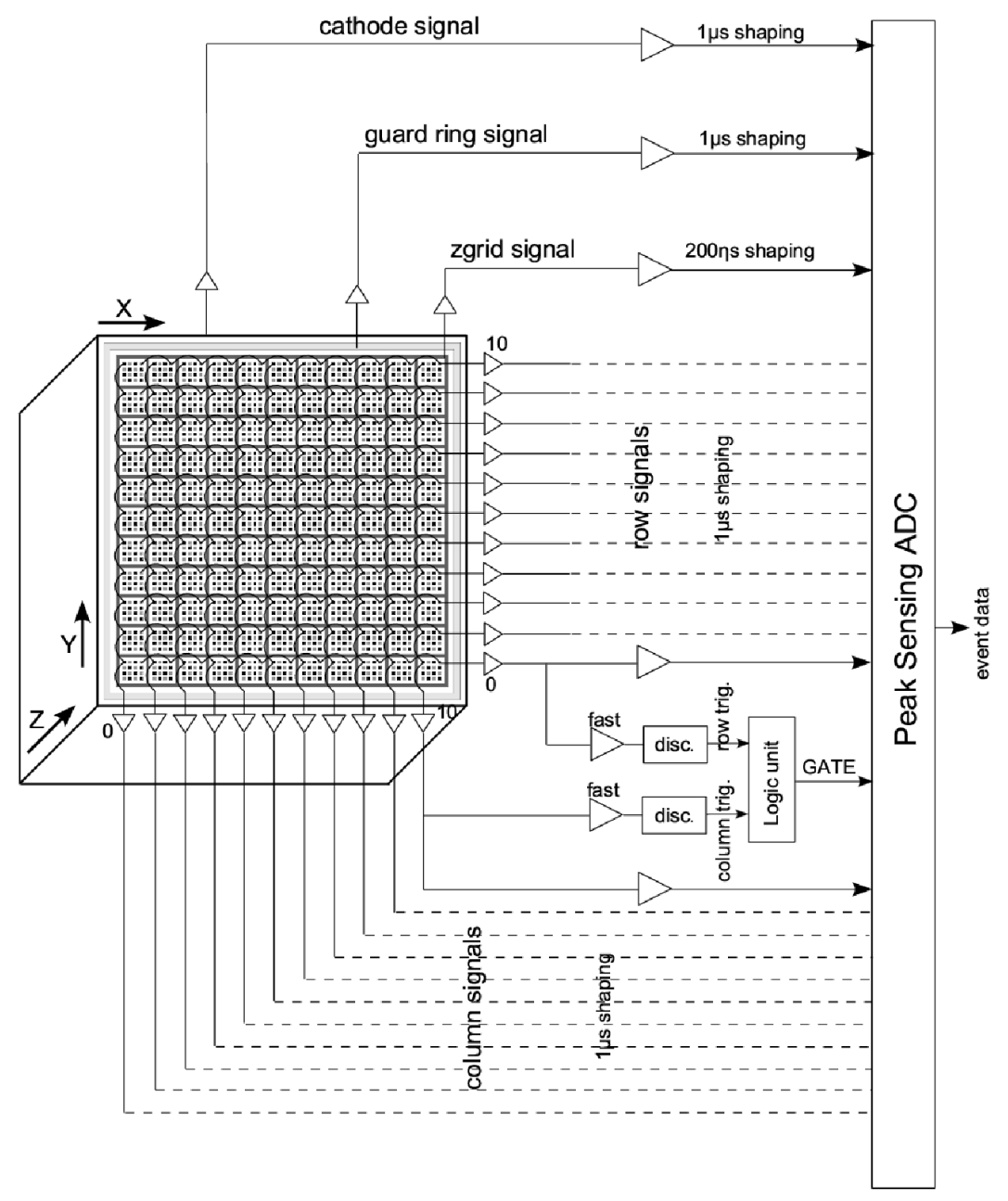

Figure 2. Experimental setup for single-sided charge-sharing CZT strip detector design.

square unit cell (expanded right) illustrates the pad interconnections. Each unit cell contains an array of closely packed anode contact pads in two groups (gray and black in this figure). The two groups are identically biased for electron charge collection but are interconnected in columns and rows in the layers of the carrier substrate. A non-collecting grid electrode surrounding each pixel, biased between pixel pad and cathode, provides a signal that can be used for measuring the depth of interaction (the $z$-coordinate). A single cathode contact on the opposite side is not shown. The principle of operation requires a sharing of charge between row and column anode contacts for each event. A more detailed description can be found in Dönmez, et al.. ${ }^{2}$

\subsection{Experimental Setup}

Figure 2 shows the signal processing setup. The detectors were inserted into a custom test board for bias and readout of the charge signals with discrete preamplifiers (eV-5093). The signal processing and data acquisition use commercially available NIM and VME electronics. Typical bias levels are $-1100 \mathrm{~V}$ for the cathode, $-30 \mathrm{~V}$ for the z-grid and $0 \mathrm{~V}$ for the rows, columns and guard ring. Each charge-collecting row and column signal is split into a shaped and a fast signal. The fast signals are used to trigger event data acquisition. When there is a signal above the discriminator level of any row or column, the peak sensing ADC is gated and the peak level of all channels, including cathode, guard ring and z-grid, is recorded. 


\section{EXPERIMENTAL RESULTS}

\subsection{Spectroscopy}

Figure 3 shows spectra from flood illumination of a detector at room temperature at energies 122 and 662 $\mathrm{keV}$. Energy resolution (FWHM) is 9.9 and $19.7 \mathrm{keV}$ at 122 and $662 \mathrm{keV}$, respectively. These single pixel spectra were constructed from the addition of maximum row and maximum column pulse heights. The photopeaks are symmetric with no significant low energy tailing that would indicate a loss of signal to the non-collecting areas of the anode surface. We believe that events for ${ }^{137} \mathrm{Cs}$ between Compton edge and photopeak events are due to the multiple Compton scattering events. ${ }^{3}$ The electronic noise is $8.0 \mathrm{keV}$ FWHM.
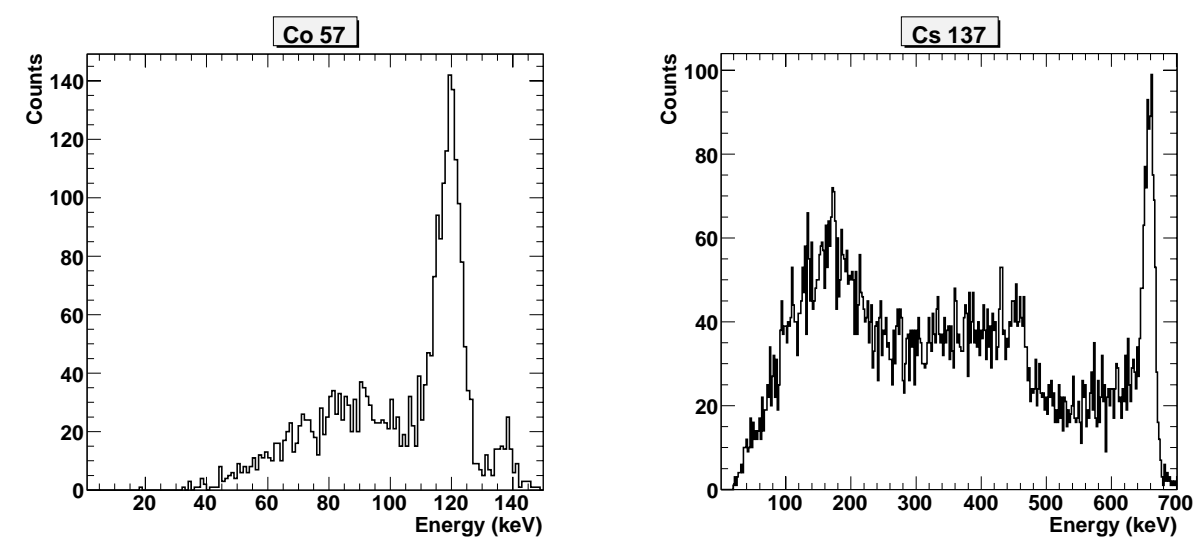

Figure 3. Spectroscopic performance of a unit cell at 122 and $662 \mathrm{keV}$.
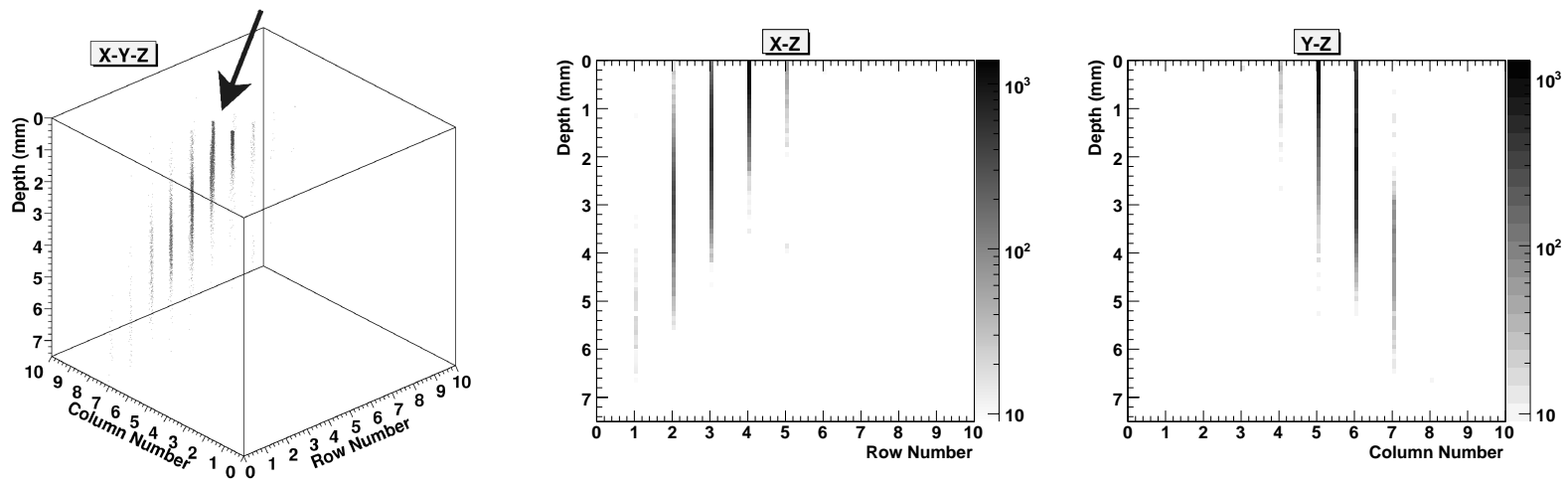

Figure 4. 3-D event locations and projections on the $x$ - $z$ and $y$ - $z$ planes for a $500 \mu \mathrm{m}$ beam of $122 \mathrm{keV}$ photons incident at $\sim 20^{\circ}$ from the normal to the cathode surface. The cathode signal is used for depth measurement. The cathode is at $z=0$.

\subsection{3-D Imaging}

3 -D imaging capability is demonstrated for $122 \mathrm{keV}$ photons using a $500 \mu \mathrm{m}$ beam spot incident on the cathode surface at $\sim 20^{\circ}$ from the $z$-axis (Figure 4 ). Photons in this beam cross several rows and columns as they pass through the detector. The row and column with the maximum signal identifies the $x$ and $y$ location for each event. There is no significant charge sharing between adjacent unit cells to permit further interpolation. The depth is measured for each event by using the ratio of the cathode-to-maximum anode signal. Spatial resolution $(1 \sigma)$ in the $z$ dimension, using the cathode signal, is less than $1 \mathrm{~mm}$. The maximum anode signal is the addition 


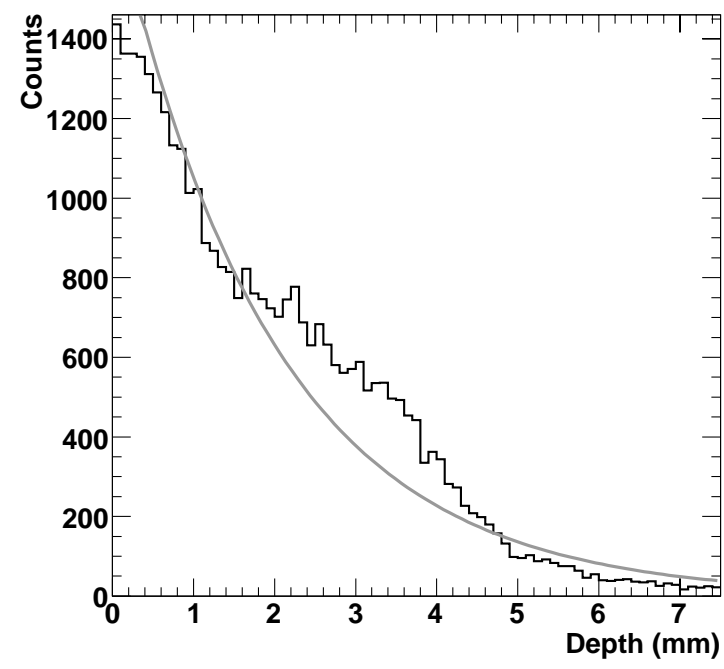

Figure 5. Calculated attenuation length for $122 \mathrm{keV}$ photons.

of the maximum row and maximum column signals. Figure 5 shows a comparison between the measured depth of interaction $(z)$ distribution for these same events and that computed from the theoretical attenuation length. The calculated attenuation length, $1.96 \pm 0.01 \mathrm{~mm}$, compares well with the theoretical value of $2.01 \mathrm{~mm}$.

\subsection{Detection Efficiency}

The intrinsic efficiency is calculated using following formula ${ }^{3}$

$$
\epsilon_{\text {int }}=\frac{\text { number of pulses recorded }}{\text { number of photons incident on detector }} \text {. }
$$

The detection efficiency is calculated for 122,356 and $662 \mathrm{keV}$ photons. For this purpose, we used ${ }^{57} \mathrm{Co}$, ${ }^{133} \mathrm{Ba}$ and ${ }^{137} \mathrm{Cs}$ sources to illuminate the entire CZT surface $12 \mathrm{~cm}$ away from the crystal surface. To eliminate edge effects, we chose a unit cell at the middle of the detector, i.e., row 5 column 5 . We found the number of pulses recorded in this cell. The number of photons incident on the detector is calculated by the following formula

$$
N_{i n}=\frac{3.7 \times 10^{4}}{4 \pi} \frac{N_{0} \times t \times A}{d^{2}}
$$

where $N_{0}$ is the activity of the radioactive source in micro Curie $(\mu \mathrm{Ci}), t$ is the duration in seconds of the experiment, $A$ is the detector area (here it is unit cell area, $0.1225^{2} \mathrm{~cm}^{2}$ ) and $d$ is the distance between source and the detector, $12 \mathrm{~cm}$. The source activity, calibrated by the manufacturer, has an error of $3 \%$. We calculated experimental efficiency using equation (1).

We used GEANT4 for simulations, which are set up so that only the unit cell is illuminated $12 \mathrm{~cm}$ away from the detector using a point source at different energies. The simulated energy threshold, $15 \mathrm{keV}$, was chosen to match that of the laboratory experiment.

Results can be seen in Table 1 and Figure 6 . The experimental efficiency values are on average $20 \%$ lower than the calculated theoretical values. The discrepancy is largely due to imaging efficiency. As reported previously, limited charge sharing among the row and column contact pads results in the identification of either the row and column but not both for $\sim 36 \%$ of the events. ${ }^{2}$ 
Table 1. Detection efficiency of single-sided charge-sharing CZT strip detector.

\begin{tabular}{ccc}
\hline \hline Energy (keV) & Experimental & Simulated \\
\hline 122 & $55.6 \pm 7.5 \%$ & $92.6 \%$ \\
356 & $24.2 \pm 4.9 \%$ & $33.1 \%$ \\
662 & $11.0 \pm 3.3 \%$ & $23.4 \%$ \\
\hline
\end{tabular}

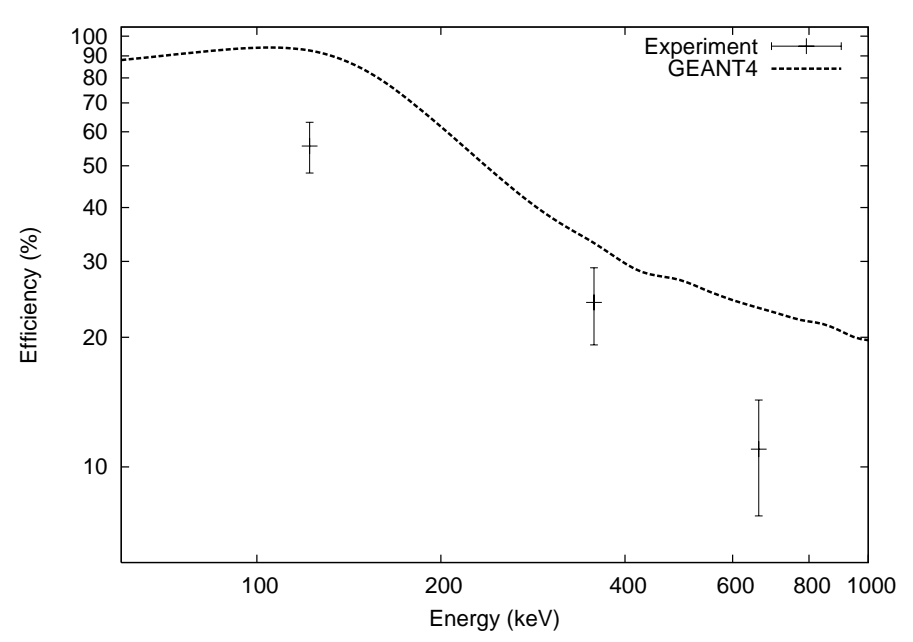

Figure 6. Detection efficiency calculations for single-sided charge-sharing CZT strip detector. GEANT4: Simulated detection efficiency. Points are the experimental detection efficiency.

\section{MULTI-HIT SIMULATIONS}

\subsection{Motivation}

Multi-hits present a measurement ambiguity for imaging with strip detectors as illustrated for a double-hit in Figure 7. Because only row and column signals are measured, interactions at points $A$ and $B$ could be interpreted as having occurred at points $C$ and $D$ unless there is some mechanism to associate the row with the column for each hit.

We have conducted simulations and measurements to determine the number and nature of multi-hits in our detectors across the energy range of interest.

\subsection{Simulation Setup}

For multi-hit simulations, we used the following procedures:

- CdZnTe detector with size $15 \times 15 \times 7.5 \mathrm{~mm}^{3}$ is placed in vacuum.

- Unit cell $\left(1.225 \mathrm{~mm}^{2}\right)$ in the center is randomly illuminated with gamma ray photons incident normal to the cathode surface from 60 to $1000 \mathrm{keV}$ in $60 \mathrm{keV}$ increments.

- GLECS $^{4}$ package for low energy Compton scattering is used in the simulations. Physical processes included are photoelectric effect, Compton scattering, gamma conversion (pair production) and Rayleigh scattering.

- All events data are written on a text file for further analysis to search for mulhi-hits. For this purpose a $\mathrm{C}++$ program was written to analyse the data file from GEANT4. 


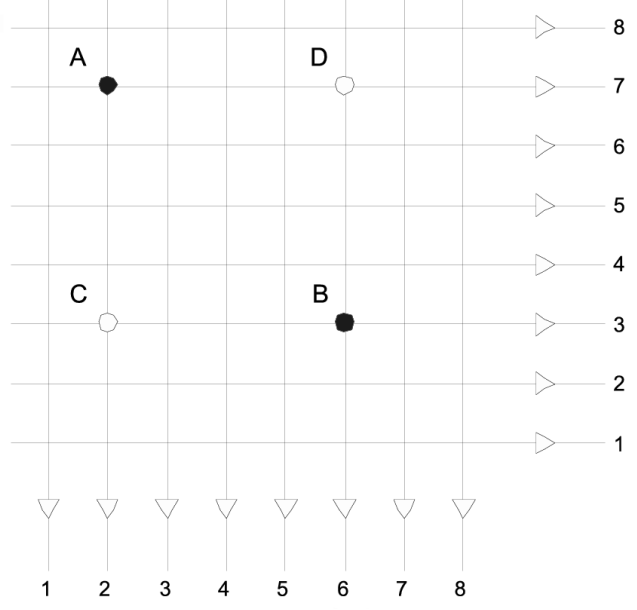

Figure 7. Illustration of how the correlation of row and column pulse height measurements can resolve multi-hit ambiguity. ${ }^{1}$

The following method is used to determine the multi-hits. For every event, we checked whether we have a Compton scattering. If the first interaction is a Compton scattering, we examine the next interaction. The event is considered as a multi-hit if it satisfies the following two conditions. First, the second interaction must be observed outside the unit cell of the first Compton scattering interaction. Second, the deposited energy at the first and second interaction sites must be larger than the $15 \mathrm{keV}$ minimum energy detectable with our detectors.

\subsection{Simulation Results}

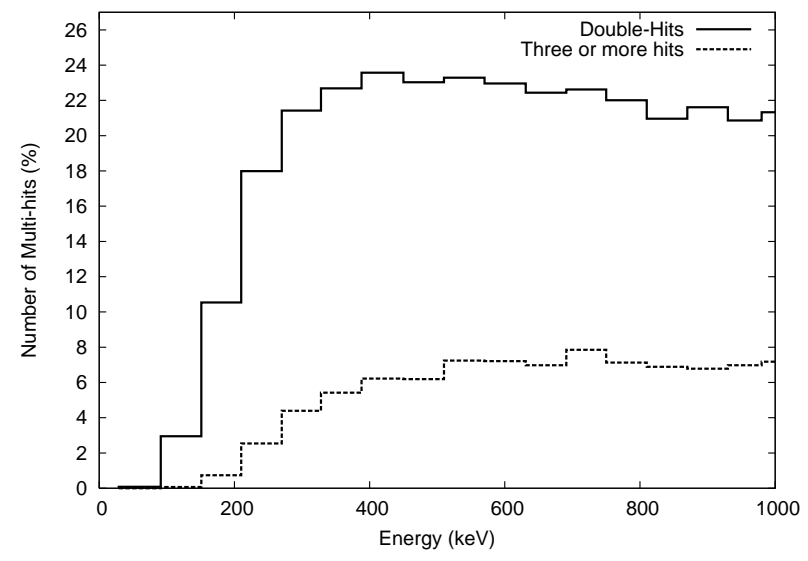

(a)

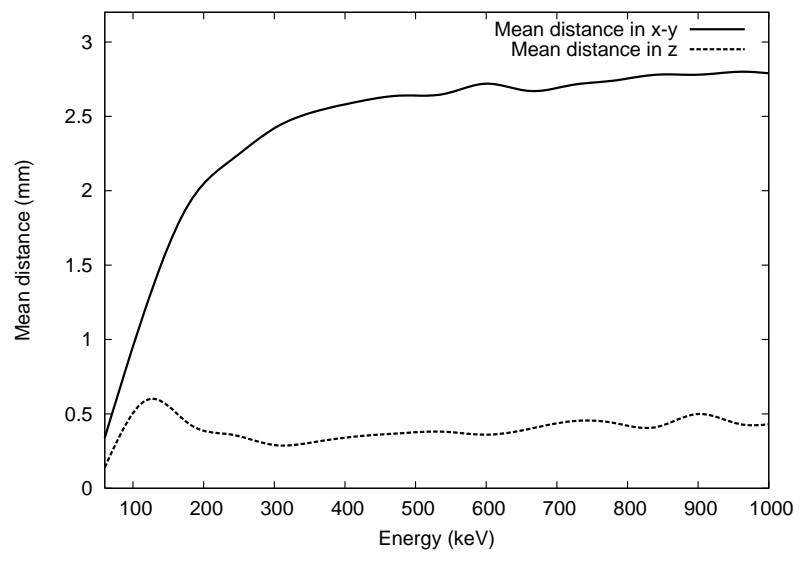

(b)

Figure 8. (a) Number of multi-hit (\%) vs. incident photon energy. Multi-hits are events with more than two interaction sites. Detector size is $15 \times 15 \times 7.5 \mathrm{~mm}^{3}$. (b) Mean distances between interaction sites for double-hit events.

We performed the series of simulations as described above. Calculated multi-hit percentages can be seen in Figure 8 (a). The number of double-hits dramatically increases at $\sim 200 \mathrm{keV}$ where Compton scattering becomes important in CZT. The number of event with three or more hits becomes significant at $\sim 300 \mathrm{keV}$. Figure 10 shows positions of the double-hit events in the lateral $(x-y)$ dimension. The mean distances between double-hit locations can be seen in Figure 8(b). 


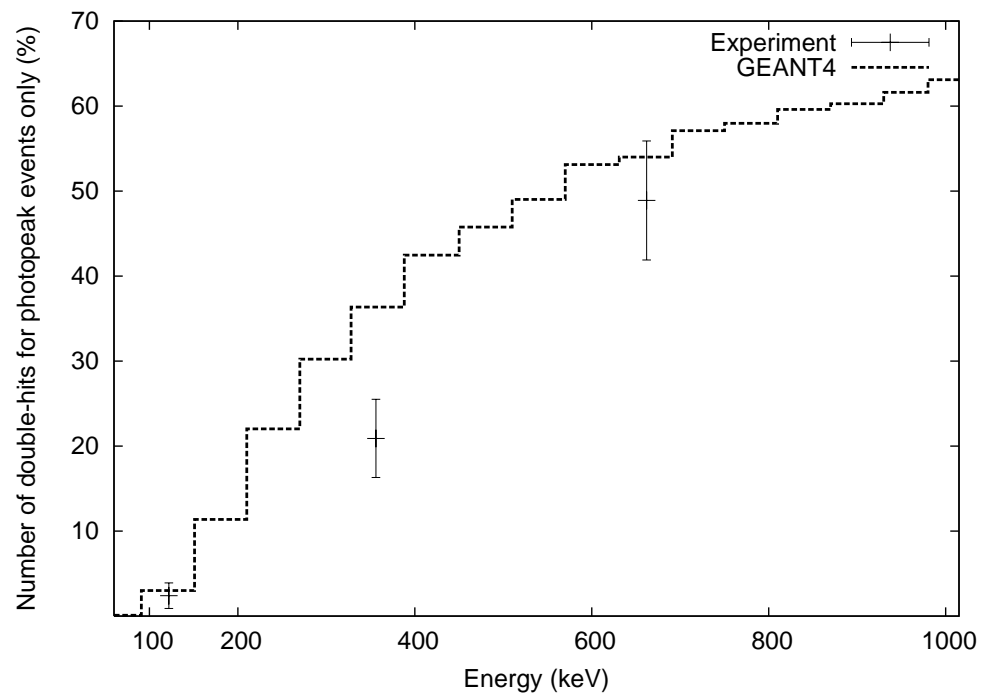

Figure 9. Comparison between experiment (points) and simulation data of double-hit for photopeak events.

To check the consistency of the simulation results, we used the flood run data of 122, 356 and $662 \mathrm{keV}$ photons from radioactive sources. To compare, we only look for the double-hit events with fully absorbed energies. The number of double-hits in the experimental data is found as follows. We select events above $15 \mathrm{keV}$ threshold at row 5 column 5, i.e. the central unit cell, as for the simulations. Then, we look at all unit cells having a signal larger than the threshold value. We sum the unit cell signal with these signals. If the summed signal resides in the photopeak, we count that event as a double-hit. The calculated double-hit percentage for photopeak events are $2.4 \%, 20.9 \%$ and $48.9 \%$ from the experiment and $3.0 \%, 36.4 \%$ and $54.0 \%$ from the simulation at 122,356 and $662 \mathrm{keV}$, respectively. The results can also be seen in Figure 9. This agreement of simulated and measured results gives confidence in the validity of the simulation tools.

\section{$122 \mathrm{keV}$}

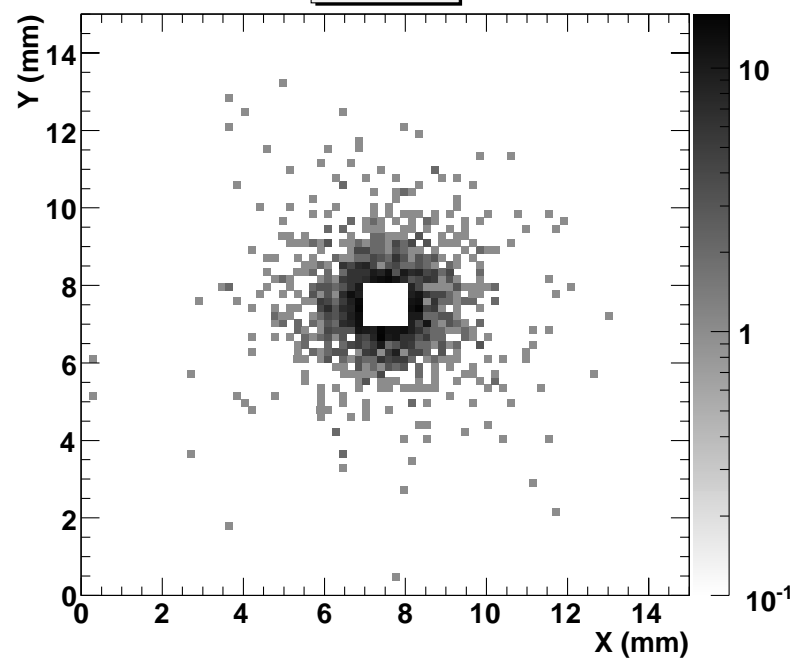

$662 \mathrm{keV}$

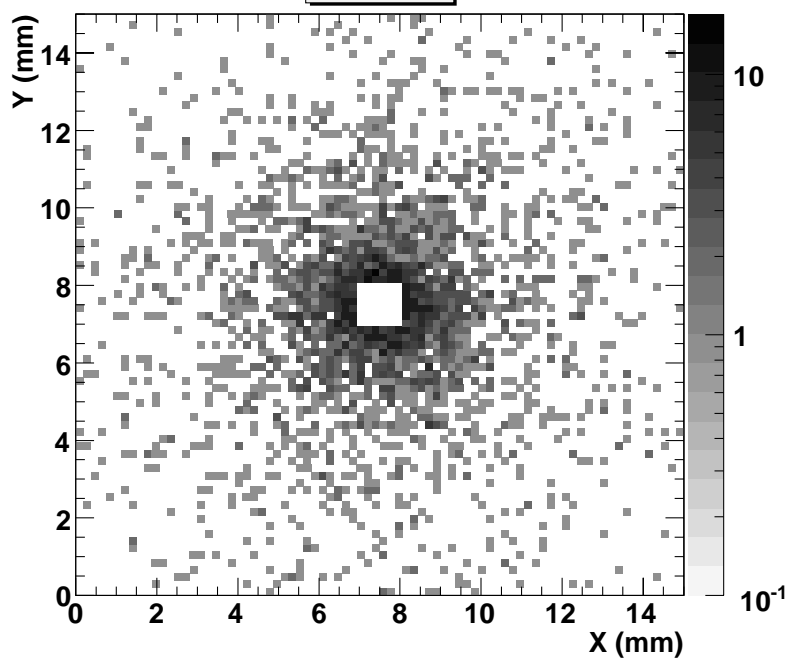

Figure 10. Simulated double-hit positions in $x-y$ at 122 and $662 \mathrm{keV}$. 


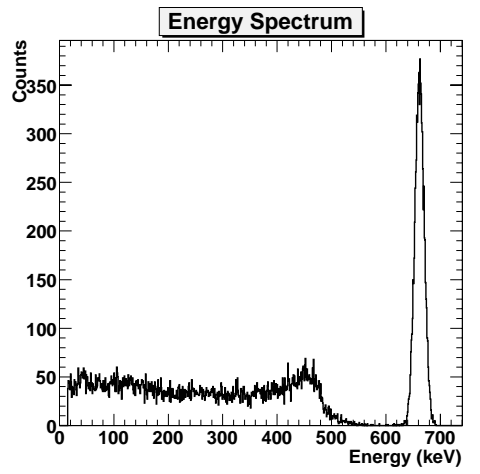

(a)

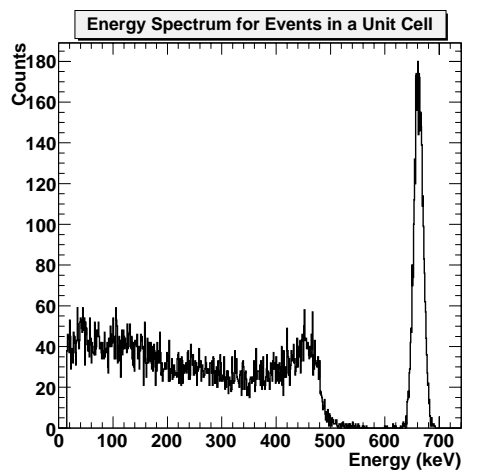

(b)

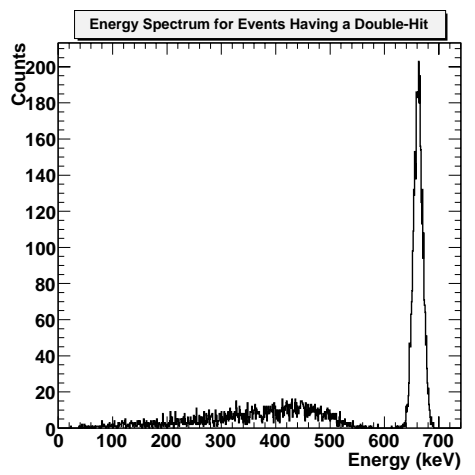

(c)

Figure 11. Simulated energy spectra including electronic noise component for all (a), for single hit (b) and for double-hit (c) events at $662 \mathrm{keV}$.

The effect of double-hits on the spectrum can be seen in Figure 11. We can see that the number of events under the photopeak for double-hit events is comparable to the number of events having only a single hit, reflecting the fact that Compton scatters are important at $662 \mathrm{keV}$.

\section{CONCLUSIONS AND FUTURE WORK}

We have continued our studies of the single-sided charge sharing CZT strip detector. These prototype devices feature $125 \mu \mathrm{m}$ anode contact pads on $225 \mu \mathrm{m}$ pitch and require signal charge to be shared among row and column pads in order to perform imaging measurements. We have demonstrated 3-D imaging capabilities equal to the row and column pitch $(1.225 \mathrm{~mm})$ in the lateral dimensions and less than $1 \mathrm{~mm}$ in the $z$ dimension. Measured detection efficiency was found to be $\sim 20 \%$ lower than the theoretical efficiency. We believe that $x-y$ imaging in efficiency as reported earlier, accounts for the discrepancy.

We also developed Monte-Carlo simulation tools based on GEANT4 (v.7.1) to study multi-hit interactions with our detectors. We observed that the number of double-hits dramatically increases at $\sim 200 \mathrm{keV}$ where Compton scattering becomes dominant in CZT. We also see that the mean distance between the first interaction and double-hit event becomes almost constant at $\sim 300 \mathrm{keV}$. We calculated and measured the percentage of double-hits for 122, 356 and $662 \mathrm{keV}$ photons. The agreement of simulated and measured results gives confidence in the validity of simulation tools.

We will continue to use these simulation tools to help us understanding the performance of our detectors. We are currently extending the simulations to include diffusion and repulsion effects. We will also develop and test prototype detectors with smaller anode contact features. We believe these next detectors will exhibit better rowcolumn charge sharing, thus improving imaging efficiency and providing a mechanism for resolving the multi-hit ambiguity.

\section{ACKNOWLEDGMENTS}

This work was supported in the USA by NASA's High Energy Astrophysics Supporting Research and Technology Program under Grant No NNG05WC25G, and in Canada by the Natural Sciences and Engineering Research Council. 


\section{REFERENCES}

1. J. R. Macri, B. Dönmez, M. Widholm, L.-A. Hamel, M. Julien, T. Narita, J. M. Ryan, and M. L. McConnell, "Single-sided CZT strip detectors," Proc. SPIE 5501, p. 208, 2004.

2. B. Dönmez, J. R. Macri, M. L. McConnell, J. M. Ryan, M. Widholm, T. Narita, and L.-A. Hamel, "Further studies of single-sided sharge-sharing CZT strip detectors," Proc. SPIE 5922, p. 62, 2005.

3. G. F. Knoll, Radiation Detection and Measurement, John Wiley \& Sons, third ed., 2000.

4. R. M. Kippen, "The GEANT low energy Compton scattering (GLECS) package for use in simulating advanced Compton telescopes," New Astronomy Reviews 48, p. 221, 2004. 